\title{
Co-Simulation Through Exchange of Time-Series Data Applied to an Energy System Model and Detailed Ground Heat Exchanger Model
}

\author{
Hauke Hirsch, Andreas Nicolai, Hans Petzold \\ Institute of Building Climatology, Technical University Dresden, Germany, \\ hauke.hirschetu-dresden.de
}

\begin{abstract}
In recent years, building energy systems have become an important area of application for Modelica. However, few components, such as ground heat exchangers, remain difficult to implement in Modelica, and thus require co-simulation with an external model. We present a method for coupling a building energy system modeled in Modelica with an external ground heat exchanger model. The so-called waveform relaxation method (WRM) realizes co-simulation by exchanging arbitrary time-series data, instead of constant/polynomial values, as currently possible with the FMI standard. This may allow for performance improvement compared to FMI under certain conditions. A major advantage of this method is the applicability to simulation tools that do not yet support FMI. First, we briefly explain the energy system model (implemented in Modelica) as well as the ground heat exchanger model (implemented in external software DELPHIN). Next, we present different implementations of the WRM coupling method and their results. Finally, we discuss the performance of WRM under certain conditions and compare it to the FMI-co-simulation approach.
\end{abstract}

Keywords: Co-Simulation, FMI, Building Simulation, HVAC System, Ground Heat Exchanger, Wave Form Relaxation

\section{Introduction}

As with the development of various libraries such as AixLib (Müller et al, 2016), IDEAS (Jorissen et al., 2018), Buildings (Wetter et al., 2014) and BuildingSystems (Nytsch-Geusen et al., 2012), Modelica has become an important tool for simulation of building energy systems. The Modelica approach is suitable for most HVAC components, as they can usually be described by differential equations with a low number of spatial dimensions. However, there remain physical problems in building energy systems, which are difficult to model in Modelica. One example are heat pump systems with borehole heat exchangers or horizontal ground heat exchangers (HGHX). These systems strongly depend on the two- or three-dimensional temperature distribution in the ground. Until now, there have been few studies addressing these problems. In (Picard and Helsen, 2014) a borefield heat exchanger model was developed, based on a TRNSYS model. The ground is discretized in radial and vertical direction and the results are close to the original model. However, for horizontal ground heat exchangers, cylindrical coordinates are not suitable and therefore a much finer spatial discretization is needed. In (Sangi and Müller, 2018) a model for horizontal slinky coil heat exchangers was developed. The soil is discretized in three dimensions and coupled to a dynamic pipe model. The model does not take into account moisture transport and freezing of soil water content and is limited to the particular slinky coil geometry. Furthermore, the presented results cover only a few days without taking into account real weather conditions such as convection and radiation at the soil surface. These effects, however, are crucial for an accurate prediction of the heat pumps efficiency at a given climate and HGHX size. This is due to the fact that electrical energy demand and heat output of heat pumps strongly depends on their source temperature.

Accurate modeling of HGHX, taking into account all relevant physical processes, requires a fine spatial discretization, usually about $<10 \mathrm{~mm}$, as shown in (Ramming 2007, Hirsch 2016). This leads to huge systems of differential equations, which need to be solved efficiently using dedicated software. DELPHIN (Grunewald 1994, Nicolai 2007) is a hygro-thermal simulation software, commonly used for component modeling in building physics. As the physical processes are similar, it can also be applied to transient heat and moisture transport in soils.

A common method for runtime coupling of different software is the FMI standard. It allows for two different coupling methods: the simulation under one common solver (ModelExchange) and coupling of independent solvers (Co-Simulation). While a number of existing simulation tools support this standard, there are still many tools, such as DELPHIN, without available FMI interface.

In the present paper, we propose an alternative approach for the coupling of independent simulation tools, 
called waveform relaxation method (WRM). WRM was originally established for solving systems of differential equations (Crow and Ilic, 1994), which can be broken into subsystems. Each subsystem is solved over the whole time domain using the time-dependent solution of another coupled subsystem. The method is repeated iteratively until convergence is reached. (Maciejewski et al, 2017) used WRM for the co-simulation of a digital power controller with fixed time step and an electrical circuit model with adaptive time stepping scheme. Both components can be integrated over the entire time span separately, which maintains their characteristics and avoids any change in their numerical implementation. This appears to be an advantage over classical co-simulation algorithms, where both solvers have to stop and communicate at a common time point, and commence integration after exchange of variables.

In our case the WRM will be conducted by coupling Modelica and DELPHIN and thus obtaining a detailed building energy supply system simulation with appropriate modeling of heat and moisture transport in the soil.

\section{Ground Heat Exchanger Model in DELPHIN}

DELPHIN is a simulation program for coupled heat, moisture and matter transport, commonly used for simulation of porous building materials. In a recent research project we implemented different soil types in DELPHIN, which enables us to model ground heat exchangers as well.
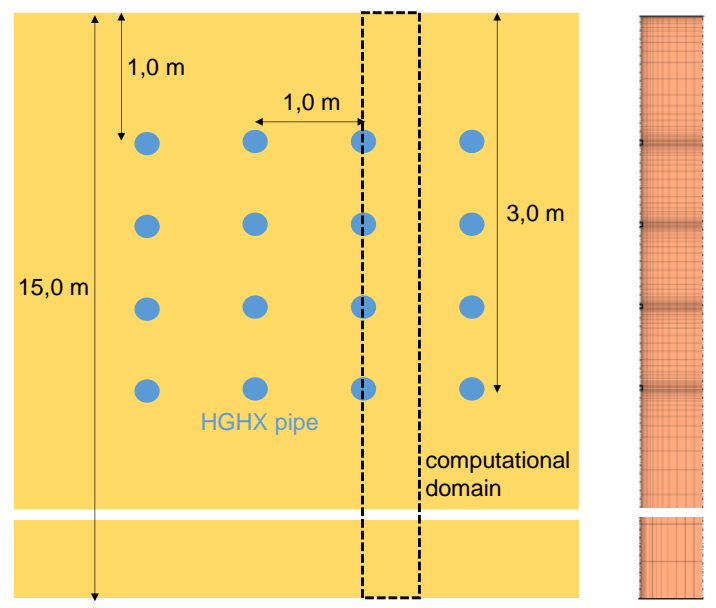

Figure 1. HGHX geometry (left) and discretization of computational domain (right)

\subsection{General Assumptions and Geometry}

The present study investigates a multi-layer horizontal ground heat exchanger shown in Figure 1, hereafter called HGHX. It consists of four horizontal layers of pipes, filled with water-glycol mixture and placed between $1 \mathrm{~m}$ and $3 \mathrm{~m}$ underneath the surface. The number of vertical rows as well as the pipe length are parameters. Most important modeling assumptions are:

- The model is reduced to the two-dimensional plane perpendicular to the HGHX tubes. Heat and moisture transport along the flow direction is neglected.

- The HGHX is considered indefinitely wide, neglecting lateral boundaries. This allows reducing the effective computational domain to the area showed in Figure 1 due to the symmetry of the temperature field.

\subsection{Spatial Discretization}

The right hand side of Figure 1 shows the discretized computational domain. A fine mesh size is used close to the soil surface and close to the pipes, while the mesh is considerably coarser further away from these boundaries. The minimum mesh size is $4 \mathrm{~mm}$ and its maximum is $70 \mathrm{~mm}$. This leads to a total number of 2944 elements. We found these mesh sizes as a good compromise between accuracy and simulation performance, based on a parameter study.

\subsection{Boundary and Initial Conditions}

At the soil volume surface, the following effects are considered:

- thermal convection

- long wave radiation

- absorption of short wave radiation

- vapor diffusion

- precipitation

The respective climate data is the test reference year TRY 2010 (Zone 4) from (Deutscher Wetterdienst, 2010). We assume constant temperature at the lower boundary, which is located at a depth of $15 \mathrm{~m}$ below the surface. This assumption is valid, since the influence of climate conditions at the surface is negligible at this depth. The lower boundary is in direct contact with water. Both lateral boundaries are considered adiabatic, due to the temperature field symmetry.

Heat exchange between the porous material and fluid inside the pipe is approximated by a special boundary condition model. It assumes steady state flow and a constant soil temperature along the pipe. The analytical solution of the pipe outlet temperature reads

$$
T_{\text {out }}=T_{s}+\left(T_{\text {in }}-T_{s}\right) \exp \left(-\frac{k A}{\dot{m} c_{p}}\right)
$$

with $T_{\text {in }}$ and $T_{s}$ being the inlet and adjacent soil temperature, $k$ refers to the heat transfer coefficient, $A$ is the outer pipe area and $\dot{m}$ and $c_{p}$ are the fluid mass flow rate and its specific heat capacity.

Before carrying out coupled simulations, we conducted an undisturbed simulation over a range of five years without any heat exchange with the HGHX to make sure quasi-steady state is reached. The resulting 
temperature and moisture fields are then used as initial values for the coupled simulations.

\section{Building Energy System Model in Modelica}

The investigated building is a small office building without domestic hot water demand. Heating energy is supplied by a heat pump and the building can be cooled through passive cooling using the HGHX. In a first step, we determined the building heating and cooling demand by simulating the building without considering energy supply and distribution. These values are used as inputs for the energy supply system. The Modelica model is built using components from the AixLib library (Müller et al, 2016) and Modelica Standard libraries and simulated using DYMOLA.

\subsection{Generic Building Model}

The considered building model was created using the tool TEASER, which allows for generation of archetype building models based on few parameters (Lauster et al, 2016). It is a small office building with an area of $400 \mathrm{~m}^{2}$ divided into the following zones:

- Office (50\%)

- Corridor $(25 \%)$

- Storage $(15 \%)$

- Meeting (4\%)

- Toilets (4\%)

- Equipment (2\%)

Each zone consists of a $4 \mathrm{~K}$ model taking into account exterior wall, interior wall, roof and indoor air. During heating season, the temperature set point is $21^{\circ} \mathrm{C}$ in the daytime and $18^{\circ} \mathrm{C}$ at night (between $7 \mathrm{pm}$ and $5 \mathrm{am}$ ) and during cooling season (between May and September) it is constant at $26^{\circ} \mathrm{C}$. This leads to a total heating demand of $13900 \mathrm{kWh} / \mathrm{a}$ and a cooling demand of $2900 \mathrm{kWh} / \mathrm{a}$.

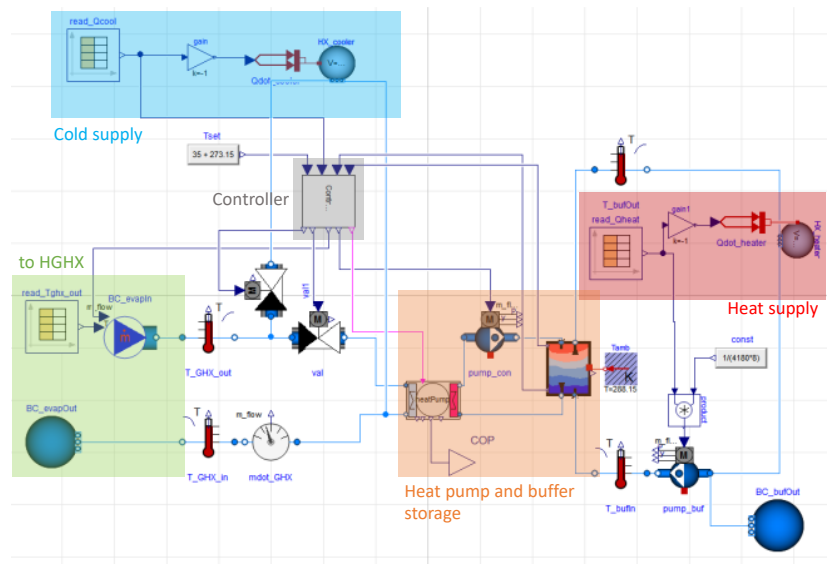

Figure 2. Energy supply system model in Modelica

\subsection{Energy Supply System Model}

The Modelica model of the energy supply system is shown in Figure 2. Basis is a brine/water heat pump, which charges a buffer storage for heat supply. The heat pump operates only in two states: on or off, as this is common practice in real systems. Its electrical energy demand and heating power are calculated through interpolation using manufacturer data tables. A two-point controller determines the heat pump operation based on the buffer storage temperature. The buffer storage model assumes one-dimensional stratification taking into account buoyancy and heat losses to the environment.

The heating and cooling demands as well as the HGHX outlet temperature (calculated in DELPHIN) are implemented as data tables. A controller determines whether the brine delivers heat (in case of heat pump operation) or is supplied with heat (in presence of cooling demand). The temperature at which the brine exits the energy supply system serves as input for the HGHX model.

\section{WRM Co-Simulation of Modelica and DELPHIN}

\subsection{Simple Approach}

The WRM differs from FMI-type co-simulation, as the coupled components are computed independently over the whole time domain of interest, rather than solving them for limited time steps. This allows the numerical solvers to run independently from each other. No changes are needed to the code of either simulation tool. For the present study, we coupled the described DELPHIN and Modelica models as shown in Figure 3.

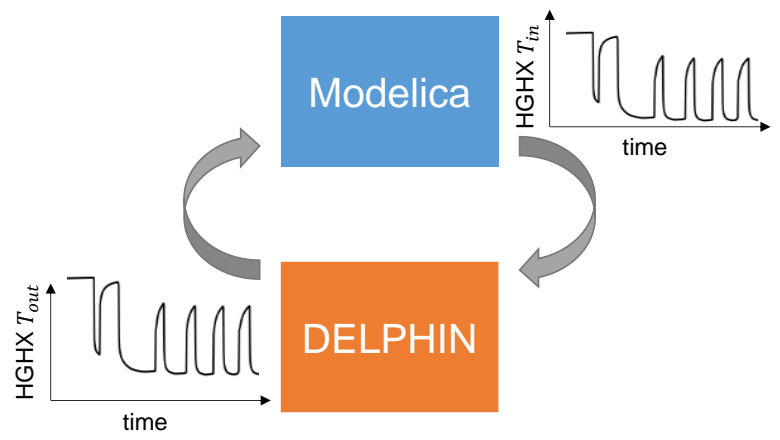

Figure 3. Simple WRM coupling scheme of Modelica and DELPHIN

First, we simulate the energy supply system over the whole time domain, typically one year, assuming a constant HGHX outlet temperature. Thus, we obtain the HGHX inlet temperature from the Modelica system model as time series for one year, which we use now as an input for the DELPHIN simulation. The obtained HGHX outlet temperature from DELPHIN is now again 


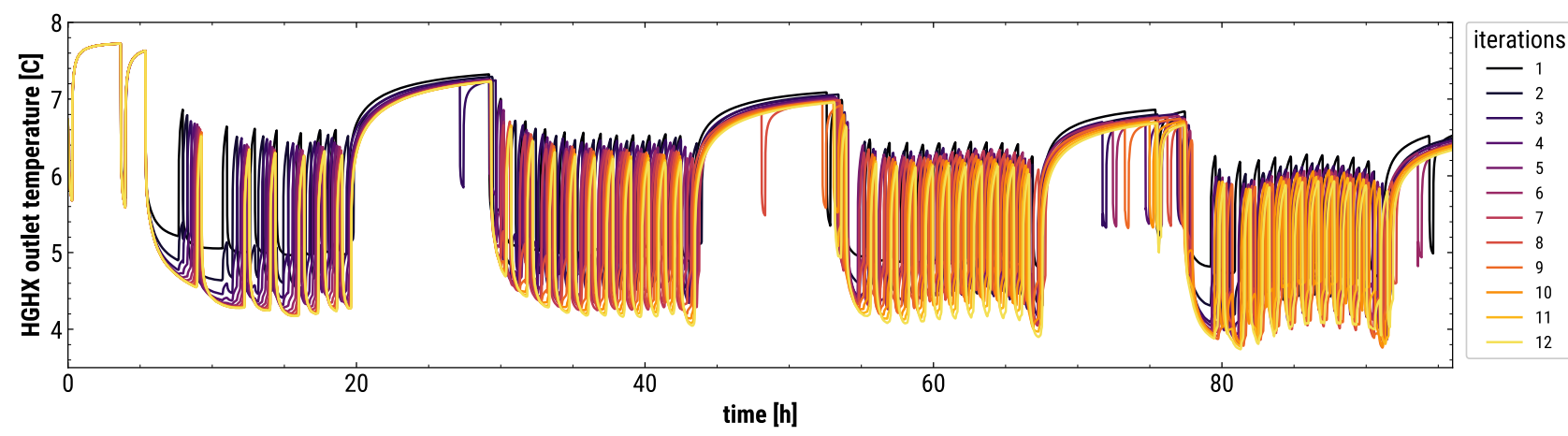

Figure 4. HGHX outlet temperature over four days using simple WRM approach

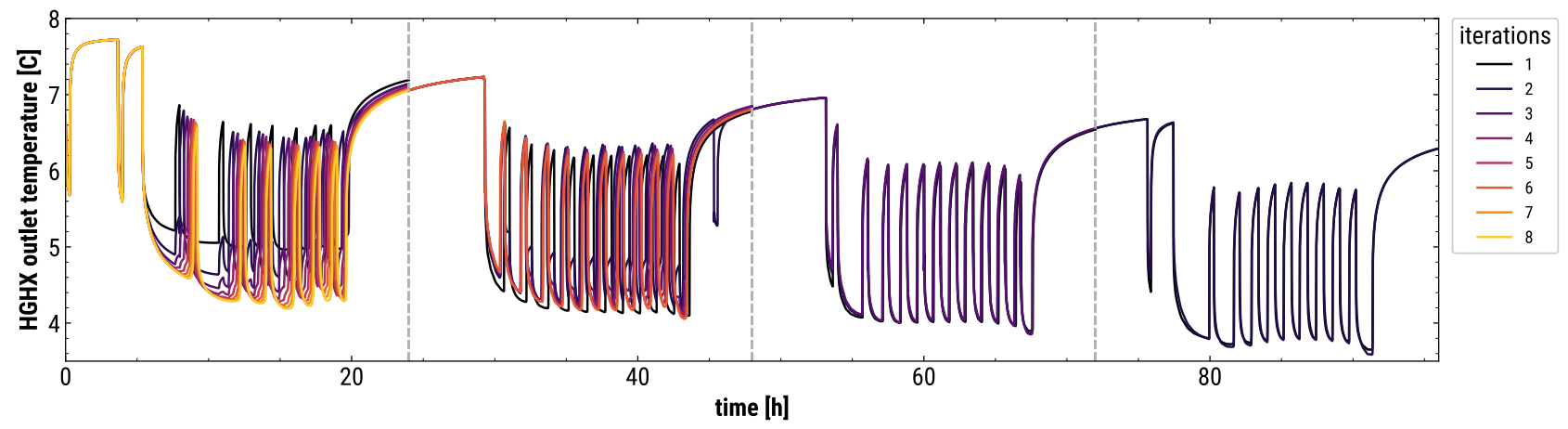

Figure 5. HGHX outlet temperature over four days using stepwise WRM approach with an interval of $24 \mathrm{~h}$

used as input for Modelica in the next iteration. The temperature may changes drastically within short periods of time, due to the intermittent behavior of the heat pump. Hence, in order to provide sufficient accuracy, both models write outputs every minute (sampling rate of the input/output signals). We implemented the method as Python script, which starts DYMOLA and DELPHIN through command line and exchanges values using txtfiles. Figure 4 depicts the HGHX outlet temperature for the first four days of the year. We ran the simulation with 12 iterations.

The heat pumps on/off characteristic controlled by a two-point controller leads to a typical behavior of the fluid temperature: When the heat pump switches on, the fluid temperature drops drastically, while when the heat pump switches off, the fluid temperature approximates the temperature of surrounding soil. However, the heat pump heating power strongly depends on its source temperature. A lower HGHX outlet temperature causes a lower heating power, and thus the heat pump needs more time to charge the buffer storage. Due to this non-linear behavior, the system converges for a limited number of iterations only within short simulation times. This can be observed in the example in Figure 4, where convergence appears to be achieved within the first day (gradually smaller differences between lines from red to yellow). However, within the following days, there is still no convergence after 12 iterations. This is due to the time shift of the operation cycles. The problem persists for the remaining simulation period. We expect the simulation to converge for all points in time after an unknown (large) number of iterations, however, simulation effort of DELPHIN is relatively high and thus, the total CPU time becomes too large to be practical usable. Hence, this simple approach to WRM co-simulation does not appear to be suitable.

\subsection{Stepwise Approach}

In order to handle the problems revealed, we implemented a new scheme, where WRM is carried out in multiple time intervals, rather than simulating over the whole time domain. As depicted in Figure 6, in each time interval, we conduct WRM as described in section 4.1. After convergence is achieved, we store the final model state. In the next time interval, both programs start, using their final states from the previous time interval and the process is repeated. This leads to a temporal decoupling and thereby clearly improves the convergence behavior of the system.

We use the root mean square norm

$$
R M S=\sqrt{\frac{1}{n} \sum_{i=1}^{n}\left(T_{\text {out }, i}-T_{\text {out }, i}^{\text {prev }}\right)^{2}}
$$

in order to determine the state of achieved convergence. Here, $T_{\text {out }}$ is the HGHX outlet temperature in the current iteration and $T_{\text {out }}^{\text {prev }}$ is the one in the previous iteration. 
It should be noted that this method requires the capability of both models to load an initial state at the beginning of a simulation and store their final state when the simulation is finished. In DYMOLA, this can be achieved using the dsin.txt, which contains the initial values of the system and the dsfinal.txt, which contains the systems final state. Both files have the same structure, so they can simply be replaced. In DELPHIN, the final state is contained in the restart file, which is created at the end of each simulation. It provides the possibility of restoring the state of the simulation model and continuing the simulation from this point in time, when DELPHIN is executed in restart mode.

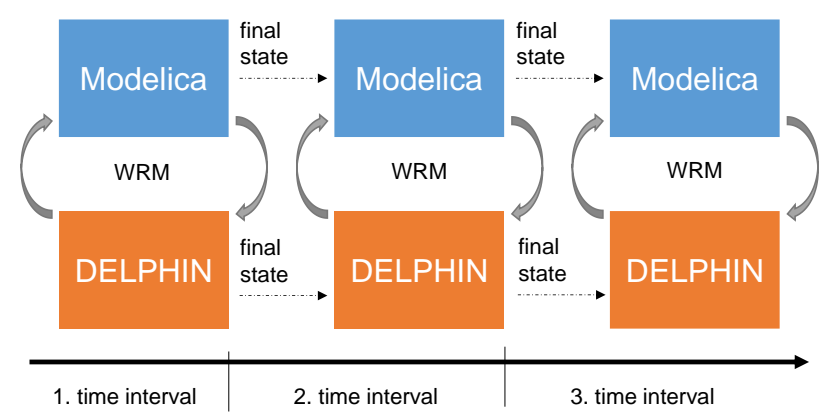

Figure 6. Stepwise WRM coupling scheme of Modelica and DELPHIN

Figure 5 shows the resulting HGHX outlet temperature with stepwise WRM using a time interval of $24 \mathrm{~h}$. In the first day, the system behaves identical to the example from chapter 4.1 and converges after eight iterations. For the next time interval, there is a distinct advantage for the iteration compared to the first time interval: since the soil temperature changes very little within one day and all other variables of Equation 1 remain unchanged, we are able to estimate the initial value of HGHX outlet temperature with relatively high accuracy.

This allows for a noticeable reduction of iterations, so that the second time interval converges within six iterations, the third time interval within three iterations and the fourth time interval within two iterations. Thus, with stepwise WRM, we achieved a total CPU time of around $150 \mathrm{~min}$ for one year simulation time, depending on chosen parameters (Core i5-7200). We consider this as practically usable, as it enables us to carry out numerous parameter studies with reasonable simulation effort.

\subsection{Assumptions and restrictions}

The presented method can be applied to the Co-Simulation of arbitrary models, with the restriction that the problem converges. Moreover, the sampling rate must be significantly below the characteristic time constant of the system, to ensure that important events are sufficiently taken into account. In our study, an operation cycle of the heat pump lasts around $30 \mathrm{~min}$, hence we used a sampling rate to $1 \mathrm{~min}$. Finally, stepwise WRM requires the capability of both models (respectively their implementation), to store their final state and use this as an initial state for the following simulation.

\section{Discussion of Performance}

\subsection{Impact of Time Interval on Performance}

The main parameter of the presented stepwise WRM approach is the time interval each WRM is conducted for. Theoretically, shorter time intervals require less iterations, which means an improvement of simulation performance. However, there is a noticeable overhead in starting a simulation model (initialization phase). Further, both Modelica and DELPHIN have variable timestep solvers based on error estimates. When started, both solvers reinitialize and start with a tiny initial time step and only gradually enlarge this time step. This constitutes a fairly large slowdown of the simulation, which in total lowers the performance for shorter time intervals.

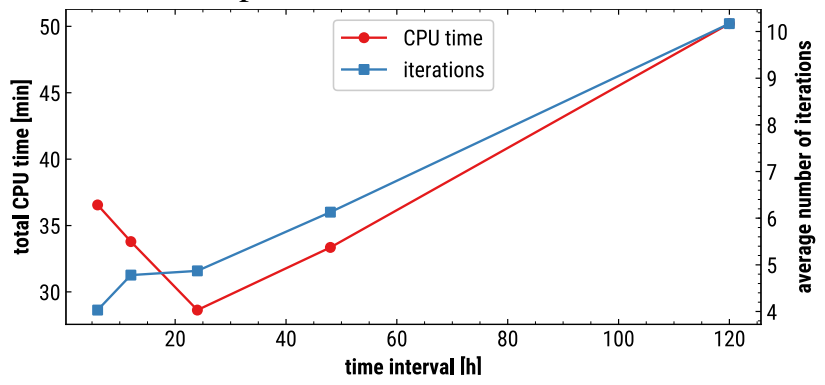

Figure 7. Total CPU time and average number of iterations using different time intervals.

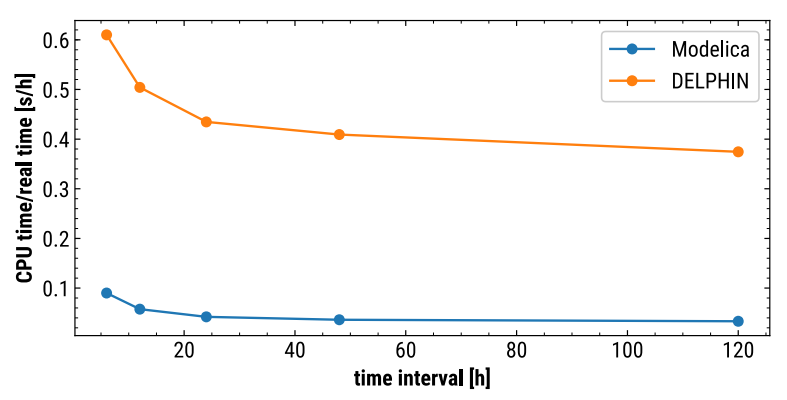

Figure 8. CPU time in relation to real time for both models using different time intervals.

We investigated both effects, by carrying out WRM co-simulations over 30 days with different time intervals ranging from $6 \mathrm{~h}$ to $120 \mathrm{~h}$. Figure 7 shows the total CPU time and the number of iterations averaged over all time intervals. As assumed, shorter time intervals require less iterations. However, the total CPU time only decreases between $120 \mathrm{~h}$ and $24 \mathrm{~h}$, while very short time intervals between $24 \mathrm{~h}$ and $6 \mathrm{~h}$ cause an increasing CPU time. This can be explained when considering the CPU time to real time relation for both models, depicted in Figure 8 . When using short time intervals both models require significantly more CPU time for the same real time, due to reasons explained. Thus, a time interval of $24 \mathrm{~h}$ appears to be a good compromise between both effects. 


\subsection{Generalization}

The WRM coupling technique is very similar to the FMI Co-Simulation with rollback as defined in version 2 of the standard. The WRM corresponds to a Gauss-Seideltype co-simulation master algorithm. However, with FMI v.2 the input and output signals are eithers constants (as supported by most FMI implementations) or polynomial functions expressed by the derivatives of a Taylor-series expansion. Complex input/output signal shapes as used in the WRM cannot be expressed by polynomials, thus communication step sizes typically need to be much smaller than those used for the WRM. Following the previous argumentation, this would incur a significant restart overhead and yield an overall slower simulation.

However, if an FMI slave were to implement a full rollback, including all variables related to the time integration and error handling, a time interval could be repeated without falling back to tiny initial step sizes (a process also known as hot-restart). In this case, utilizing FMI Co-Simulation for a WRM-type signal input/output handling can be an interesting approach with potentially much higher performance, since the FMI rollback feature can help avoid the excessive overhead in restarting/reinitializing simulations. Currently, only the SimulationX Modelica environment has documented such a rollback functionality.

\section{Summary}

We investigated the application of the waveform relaxation method (WRM) for a co-simulation between Modelica and an external software. Therefore, we used the example of a building energy system simulation (Modelica), which was coupled to a ground heat exchanger model (DELPHIN). We showed that the non-linear behavior of the Modelica model causes convergence problems when a simple WRM approach is used. The simulation converges only for short time domains within reasonable number of iterations. In order to tackle that problem, we introduced a stepwise approach, where WRM is carried out in limited time intervals. This provides temporal decoupling, so that the simulation converges with noticeably less iterations.

We revealed that the simulation performance of the stepwise WRM strongly depends on the chosen time interval. While long time intervals cause many iterations, short time intervals increase the overhead of restarting/reinitializing the individual simulations. Eventually, a comparison to FMI Co-Simulation has been undertaken. We propose that the possibility of using complex input/output-signals in FMI-coupling may yield a significant performance improvement, in particular if used with a full rollback, where excessive overhead due to reinitializing is avoided.

\section{Acknowledgements}

We gratefully acknowledge the support and funding received from the German Federal Ministery for Economic Affairs and Energy in the research project "EnEff:Wärme - Erdeisspeicher und oberflächennahe Geothermie" \#03ET1382A.

\section{References}

M.L. Crow and M.D. Ilic. The Waveform Relaxation method for systems of differential/algebraic equations. Mathematical and Computer Modelling, Volume 19, Issue 12, June 1994, Pages 67-84.

Deutscher Wetterdienst. Testreferenzjahre von Deutschland für mittlere und extreme Witterungsverhältnisse TRY. DWD, Offenbach am Main, Deutschland, 2010.

John Grunewald. Diffusiver und konvektiver Stoff- und Energietransport in kapillarporösen Baustoffen. $\mathrm{PhD}$ thesis, Dresden, TU Dresden, 1997.

F. Jorissen, G. Reynders, R. Baetens, D. Picard, D. Saelens, and L. Helsen. Implementation and Verification of the IDEAS Building Energy Simulation Library. Journal of Building Performance Simulation, 11 (6), 669-688, doi: 10.1080/19401493.2018.1428361, 2018.

Hauke Hirsch, Fabian Rüsing, Gunter Rockendorf. Modellierung oberflächennaher Erdwärmeübertrager für Systemsimulationen in TRNSYS. BauSim 2016, Dresden, Germany. 2016.

M. Lauster et al. Design-Driven Parameterization of Reduced Order Models Using Archetype Buildings with TEASER. BauSIM 2016, Dresden, Germany. 2016.

M. Maciejewski, I. Cortes Garcia, S. Schöps, B. Auchmann, L. Bortot, M. Prioli, and A.P. Verweij. Application of the Waveform Relaxation Technique to the Co-Simulation of Power Converter Controller and Electrical Circuit Models. 22nd International Conference on Methods and Models in Automation and Robotics (MMAR). Miedzyzdroje, 2017. pp. 837-842. doi: 10.1109/MMAR.2017.8046937

D. Müller, M. Lauster, A. Constantin, M. Fuchs, P. Remmen. AixLib - An Open-Source Modelica Library within the IEA-EBC Annex 60 Framework. BauSIM 2016, Dresden, Germany. 2016.

Andreas Nicolai. Modeling and numerical simulation of salt transport and phase transitions in unsaturated porous building materials. PhD thesis, New York, Syracuse University, 2007.

Christoph Nytsch-Geusen; Jörg Huber; Manuel Ljubijankic; Jörg Rädler. Modelica BuildingSystems - Eine Modellbibliothek zur Simulation komplexer energietechnischer Gebäudesysteme. BAUSIM 2012 IBPSA Germany, 26.-28. September. Conference Proceedings. Berlin, 2012.

Damien Picard and Lieve Helsen. Advanced Hybrid Model for Borefield Heat Exchanger Performance Evaluation, an Implementation in Modelica. $10^{\text {th }}$ International Modelica Conference, March 10-12, 2014, Lund, Sweden. doi: 10.3384/ecp14096857\}

Klaus Ramming. Bewertung und Optimierung oberflächennaher Erdwärmekollektoren für verschiedene Lastfälle. $\mathrm{PhD}$ thesis, Dresden, TU Dresden, 2007. 
Roozbeh Sangi and Dirk Müller. Dynamic modelling and simulation of a slinky-coil horizontal ground heat exchanger using Modelica. Journal of Building Engineering 16 (2018) 159-168. https://doi.org/10.1016/j.jobe. 2018.01.005.

Michael Wetter, Wangda Zuo, Thierry S. Nouidui and Xiufeng Pang. Modelica Buildings library. Journal of Building Performance Simulation, 7(4): 253-270, 2014. 
